\title{
Quantum crystallography and spintronic materials
}

\author{
Piero Macchi \\ Department of Chemistry, Materials and Chemical Engineering, Polytechnic of Milan, via Mancinelli 7, 20131, Milan, Italy \\ piero.macchi@polimi.it
}

Quantum information theory is developing at a rapid pace and one can easily envisage a bright future, demonstrated by the growing number of quantum computing simulations offered at large scale computation facilities and by the construction of the first prototypes of quantum computers.

In this area, the contribution of crystallography, and especially of quantum crystallography, is seamlessly vital, because the inner mechanism at hearth in the transmission of signal is tightly connected with the chemical bonding, the electron charge and spin density distribution, and, ultimately, the wavefunction.

Quantum Crystallography $[1,2,3]$ deals, in fact, with the application of quantum theory in crystallography. Among the various quantities investigated within this field, the electron delocalization and the spin electron density play a fundamental role in spintronic materials.

Over the years, several theoretical analyses emerged that can be applied to computed as well as to experimental electron densities. Moreover, the modelling techniques enable the reconstruction of many quantities from ever more precise and sophisticated experiments.

In this lecture, novel spintronic materials, such as magnetic coordination polymers [4, 5], will be discussed, within the framework of recent quantum crystallographic studies. The role of the linkers in the magnetic exchange is not fully clear yet. A combinatorial approach, including studies on materials at high pressure shed light on the subtleties of exchange mechanisms.

[1] A. Genoni et al. Chemistry, Eur. J., 2018, 24, 10881-10905.

[2] P. Macchi Cryst. Rev. 2020, 26, 209-268.

[3] P. Macchi Quantum Crystallography: Fundamentals and applications 2022, De Gruyter to be published.

[4] Kubus, M., Lanza, A., Scatena, R., Dos Santos, LHR., Wehinger, B., Casati, N., Fioka, C., Keller, L., Macchi, P., Rüegg, C., Krämer, KW. (2018) Inorg. Chem. 57, 4934-4943.

[5] R. Scatena, R. D. Johnson, P. Manuel, P. Macchi, J. Mater. Chem. C 2020, 8, 12840-12847. 